\title{
Coming Unbound: Disrupting Automatic Integration of Synesthetic Color and Graphemes by Transcranial Magnetic Stimulation of the Right Parietal Lobe
}

\author{
Michael Esterman $^{1,2}$, Timothy Verstynen ${ }^{1}$, Richard B. Ivry ${ }^{1}$, \\ and Lynn C. Robertson ${ }^{1,2}$
}

\begin{abstract}
In some individuals, a visually presented letter or number automatically evokes the perception of a specific color, an experience known as color-grapheme synesthesia. It has been suggested that parietal binding mechanisms play a role in the phenomenon. We used a noninvasive stimulation technique, transcranial magnetic stimulation (TMS), to determine whether the posterior parietal lobe is critical for the integration of color and shape in color-grapheme synesthesia, as it appears to be
\end{abstract}

\section{INTRODUCTION}

In color-grapheme synesthesia, specific graphemes are automatically seen in specific colors. For example, the letter " $A$ " is always perceived in a particular shade of red. This unusual variety of color-form binding has been well characterized behaviorally. However, the neural correlates of synesthesia are not well understood (Rich \& Mattingley, 2002). Although functional magnetic resonance imaging (fMRI) has shown that increased activity in extrastriate cortex is associated with the perception of synesthetic photisms (Sperling, Prvulovic, Linden, Singer, \& Stirn, 2006; Hubbard, Arman, Ramachandran, \& Boynton, 2005), other brain imaging studies of color-grapheme synesthesia have shown neural correlates outside of the ventral occipital cortex in the posterior parietal cortex (PPC). Rich et al. (2003) found activation in the right PPC (at the junction of the occipital cortex) during synesthetic color-grapheme perception, an area implicated in normal binding of color and shape (Donner et al., 2002). Others have found activation in the intraparietal sulcus (IPS) associated with color-grapheme synesthesia, both in the left hemisphere (Weiss, Zilles, \& Fink, 2005) and bilaterally (Elias, Saucier, Hardie, \& Sarty, 2003).

The PPC has already been associated with color-form binding in normal perception using tasks that involve con-

\footnotetext{
${ }^{1}$ University of California, Berkeley, ${ }^{2}$ Veterans Administration Medical Center, Martinez, CA
}

for normal color-shape binding. Using a color-naming task with colored letters that were either congruent or incongruent with the synesthetic photism, we demonstrate that inhibition of the right posterior parietal lobe with repetitive TMS transiently attenuates synesthetic binding. These findings suggest that synesthesia (the induction of color from shape) relies on similar mechanisms as found in normal perception (where the perception of color is induced by wavelength).

junction visual search (Donner et al., 2002; Ashbridge, Walsh, \& Cowey, 1997). In addition, patients with bilateral PPC lesions have difficulty in correctly conjoining shape and color. For example, R.M., a patient with Balint's syndrome, makes frequent illusory conjunctions in free viewing conditions (Friedman-Hill, Robertson, \& Treisman, 1995). Together, these findings suggest that although synesthesia is an atypical integration of color and shape, it may rely on similar mechanisms to normal perceptual feature binding (Cohen-Kadosh \& Henik, 2006; Sagiv, Heer, \& Robertson, 2006; Robertson, 2003; Grossenbacher \& Lovelace, 2001).

Further evidence that parietal mechanisms may be involved in synesthesia come from behavioral studies demonstrating that binding of the synesthetic photism requires awareness of the inducing grapheme and is modulated by whether the grapheme is within the spotlight of attention (Sagiv et al., 2006; Palmeri, Blake, Marois, Flanery, \& Whetsell, 2002; Mattingley, Rich, Yelland, \& Bradshaw, 2001). The idea that synesthesia arises from operations of an attentional binding mechanism is challenged by findings indicating preattentive activation of synesthetic percepts (Smilek, Dixon, \& Merikle, 2004; Ramachandran \& Hubbard, 2001). These contradictory results may be explained in part by individual differences between synesthetes (Hubbard \& Ramachandran, 2005).

We sought to test the hypothesis that parietal binding mechanisms that are necessary for normal perception also play a role in color-grapheme synesthesia. To this 
end, we used a color-naming task (Mattingley et al., 2001; Dixon, Smilek, Cudahy, \& Merikle, 2000). On each trial, a colored letter was presented in one of three colors and the participant pressed a key to indicate the color. The color was either congruent or incongruent with the synesthetic photism (Figure 1B). Synesthetic facilitation occurs when responses to letters presented in their synesthetic color (congruent condition) are faster than baseline responses (neutral characters that do not evoke a synesthetic photism). Synesthetic interference occurs when responses to letters presented in colors that are incongruent with their synesthetic photism (incongruent condition) are slower than baseline. This task was performed following transcranial magnetic stimulation (TMS) of the left or right parietal cortex. This method allowed us to investigate whether transient inhibition of the parietal cortex influenced synesthetic induction of color by shape. Specifically, would parietal stimulation reduce any photism-induced facilitation or interference? Such changes would be consistent with this region contributing to binding of color and shape in synesthesia.

We targeted the angular gyrus at the junction of the posterior IPS and transverse occipital sulcus (IPS/TOS), a region associated with color-form binding in normal perception (Donner et al., 2002). In addition, activation in the right IPS/TOS has been observed during synesthetic color-grapheme perception (Rich et al., 2003), suggesting a correspondence between normal and synesthetic binding of color and form. In separate sessions, stimulation was applied over this parietal region of the right and left hemisphere. In another session, repetitive
TMS (rTMS) was applied over the primary visual cortex (V1) to test for the effects of generalized brain stimulation on responses in this task.

\section{METHODS}

\section{Participants}

All procedures for this experiment were approved by the local ethical review board at University of California at Berkeley. We tested two color-grapheme synesthetes recruited from the local population at the university: C.P. (27 years old) and E.F. (22 years old), both righthanded women. Both report having a unique set of alphanumeric-color associations (see Figure 1A) that are stable over time. Both reported their colors were projected in the external world and appeared as a "property" of the inducing character, which was also in the "mind's eye." Both synesthetes would be classified as "projectors" (Sagiv et al., 2006; Dixon, Smilek, \& Merikle, 2004). It has been shown in a prior behavioral study that attention modulates the extent of C.P.'s synesthetic experience (Sagiv et al., 2006). Moreover, her experiences were previously associated with modest activation of lower visual areas, leading to her classification as a "higher synesthete" (see participant C.H.P. in Hubbard et al., 2005).

We did not test participants with normal perception in this study because they have no synesthetic photism to influence color naming (see behavioral procedures described below) and training controls with colorgrapheme associations would not replicate the experience of synesthesia.
Figure 1. Behavioral and TMS procedure for participant E.F. (A) Synesthetic alphabet. (B) Individualized experimental conditions. The task was to name the stimulus color. In the congruent condition, the stimulus color matched the synesthetic color. In the incongruent condition, the stimulus color did not match the synesthetic color. Neutral characters did not evoke a synesthetic color. (C) Target stimulation site (right IPS TOS) shown on coronal and axial slices, plus a 3-D reconstruction of the anatomical image for E.F. Target location is shown in red, center of magnetic coil is shown as green spheres in the 3-D image, and estimated pulse and orthogonal trajectories are shown as yellow lines.
A

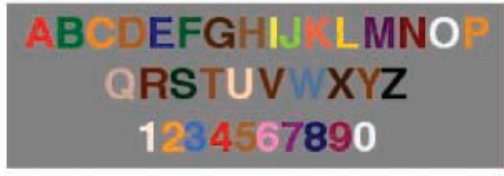

B

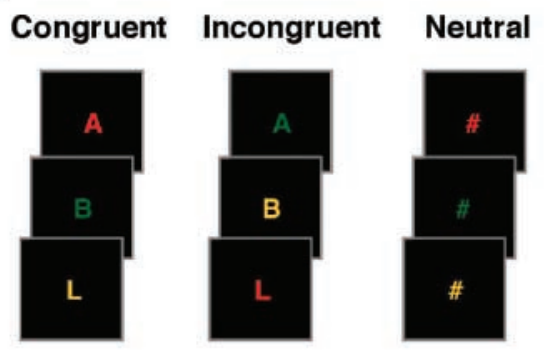

C
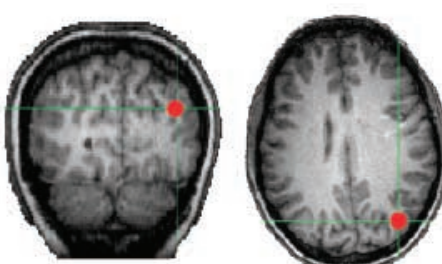

-IPS/TOS

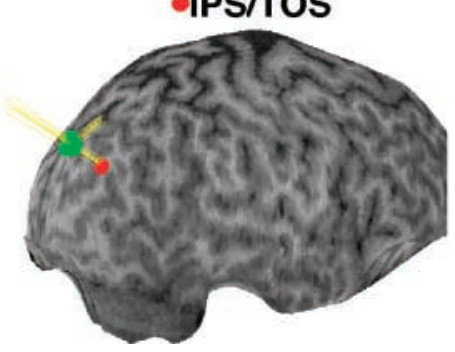




\section{Behavioral Procedure}

Before testing, we estimated the specific RGB screen values for each letter of each participant's synesthetic alphabet. They were seated comfortably $\sim 30 \mathrm{~cm}$ in front of a 19-in. CRT monitor ( $70 \mathrm{~Hz}$ refresh rate). An alphanumeric symbol was presented on the screen and the participant was instructed to adjust the RGB values until the color on the screen matched their synesthetic photism. For testing, we chose those letters that evoked the most red, green, and yellow color associations, respectively. Each shade of red, green, and yellow was chosen to closely match the synesthetic photism.

To quantify each participant's synesthesia we used a color-naming task. A series of colored single letters (red, green, or yellow; see above) were presented, each for $1000 \mathrm{msec}$, in a color that was either congruent (congruent trials) or incongruent (incongruent trials) with the synesthetic photism, or a symbol ("\#") was presented that did not evoke a synesthetic photism (neutral trials; see Figure 1B for examples). Participants pressed a button with their right hand indicating the true color of the letter as quickly as possible while trying to ignore the synesthetic photism. Reaction time to manually respond to the screen color was recorded as the difference between the onset of the letter and the subsequent keypress. There was no deadline to respond. Following a response, there was a $1000 \mathrm{msec}$ intertrial interval. Stimuli were presented with PsychLab software (Teren Gum, Boston, VA), and recorded with a CMU button box (three buttons pressed with three different fingers).

Each block consisted of 120 trials (48 congruent, 48 incongruent, and 24 neutral, representing all combinations of colors and characters). After each stimulation epoch (sham rTMS or real rTMS; see below), participants were tested on two blocks of trials: an early block (1-5 min poststimulation) and a late block (6-10 min poststimulation). Order of sham and real TMS was counterbalanced for each participant. Each sham and real TMS epoch was performed twice in each session, alternating between stimulation types. Separate sessions were done for each target region (right parietal, left parietal, V1). For each session, 960 trials were collected per participant.

\section{Transcranial Magnetic Stimulation}

We first obtained high-resolution anatomical MRIs for each participant. The images for C.P. were acquired in a previous study using a MPRAGE sequence on a $1.5 \mathrm{~T}$ Siemens Vision scanner at the University of California, San Diego $(1 \times 1 \times 1$ isotropic voxels, 180 slices $)$. The images for E.F. were acquired using a MPFLASH protocol on a Varian INNOVA $4 \mathrm{~T}$ system at the University of California, Berkeley $(2 \times 2 \times 2$ isotropic voxels, 128 slices). We identified the target regions for TMS stimulation from each participant's MRI (left and right IPS/
TOS and V1). The voxel location was marked on a skullstripped reconstructed image.

The scalp location was determined by using a stereotaxic localization system (Brainsight, Rogue-Research Inc., Montreal, Canada). Coil position over the target regions was monitored online during the stimulation epochs. In addition, trajectory estimates of the TMS pulse were estimated intermittently throughout recording (green dots and yellow lines in Figure 1C). Although we chose our region of interest (ROI) based on each individual's anatomy, we estimated the Talairach coordinates of the right IPS/TOS site as $(30,-74,32)$, close to the area associated with feature binding $(22,-71,27)$ in Donner et al. (2002). Talairach space is primarily driven by the need to standardize across individuals and assumes a standard sulcal and gyral geometry. By nature, it is insensitive to individual differences in brain structure. The locations in the present study were identified by using the pattern of gross anatomical landmarks of the IPS, angular gyrus, and TOS. Thus, this coordinate is only an estimate of our stimulation location.

rTMS was performed by using an iron-cored figure-8 coil (NeoTonus Inc., Marietta, GA; see Epstein \& Davey, 2002). Before each session, the participant's active motor threshold was determined as the point at which four to six visible twitches of the thumb were detected following 10 pulses over the motor cortex while the thumb and index finger were held together in a pinchlike posture. C.P.'s threshold was between 35\% and 38\% maximum stimulator output, whereas E.F.'s threshold was consistent at $45 \%$. Stimulation was then set to $115 \%$ of motor threshold for the remainder of the experiment. Each stimulation epoch consisted of 480 consecutive pulses that were delivered at a rate of $1 \mathrm{~Hz}$ ( $8 \mathrm{~min})$. This low-frequency design causes a transient inhibition of the underlying cortex, with the duration of the effect roughly equal to the duration of the stimulation at $1 \mathrm{~Hz}$ (Pascual-Leone et al., 1998). Thus, maximal behavioral effects of TMS were expected during the early block (minutes $1-5$ post stimulation) diminishing during the late block (minutes 6-10 post stimulation). During rTMS blocks, the coil was oriented to deliver stimulation directly to the target cortical location. For sham control rTMS blocks, the coil was oriented $90^{\circ}$ away from the scalp so that no pulses perturbed underlying neural tissue. The subjects were naive to whether they were receiving sham or real stimulation.

\section{Data Analysis}

Given the small sample size and the propensity for large between-subjects variability with synesthesia, we adopted a strong within-subject method to analyze the stimulation effects. The TMS effects were statistically analyzed by using randomization methods on a singlesubject basis (e.g., bootstrapping; see Manly, 1997). All analyses excluded any reaction times greater than four 
standard deviations from the mean (less than 1\% of trials for each participant). We first recorded the interference effect for each of the six conditions (3 regions $\times 2$ rTMS/ sham epochs) by subtracting reaction times to incongruent trials from neutral trials. A "true" TMS effect score $\left(X_{\mathrm{t}}\right)$ was determined by subtracting the interference effect observed following sham stimulation from the effect found after real rTMS. A score of zero indicates no change in interference following rTMS, a positive score indicates increased interference following rTMS, and a negative score represents a decreased interference effect after rTMS. We then randomly recategorized reaction times between neutral and incongruent trials and recalculated a new interference effect. This was done separately for rTMS and sham trials, following which a new TMS effect score was calculated. This scrambling, recategorization, and subtraction process was repeated for 10,000 iterations to produce a hypothetical probability distribution of interference effects that would occur simply by chance. Figure $2 \mathrm{~A}$ shows an example of a random distribution of interference effects for C.P. following right-hemisphere stimulation. The dashed line represents her true TMS effect. These distributions closely resembled normal distributions, as verified using probability plots (Figure 2B; Chambers, Cleveland, Kleiner, \& Tukey, 1983).

The probability of getting the true observed effects by chance $(p)$ can then be directly calculated as:

$$
p=\Sigma\left(X_{\mathrm{r}}>X_{\mathrm{t}}\right)
$$

where $X_{\mathrm{r}}$ is the array of values pulled from the randomized null distribution.

\section{RESULTS AND DISCUSSION}

Following sham rTMS, both participants showed synesthetic interference in all six blocks (E.F.: mean = $51 \mathrm{msec}$, range 32-90 msec; C.P.: mean = $31 \mathrm{msec}$; range, $19-48 \mathrm{msec})$. In contrast, rTMS of the right parietal ROI significantly attenuated interference for both participants during the early test block (by $54 \mathrm{msec}$ for E.F. and $48 \mathrm{msec}$ for C.P., $p<.01$; see Figure 3). For E.F., interference returned to baseline levels in the late block (61 msec); however, C.P.'s scores were still attenuated (10 msec), suggesting the effects of rTMS had not completely worn off. C.P.'s responses were generally faster overall following real rTMS, likely due to generalized arousal. More importantly, the attenuation of interference seen after right IPS/TOS stimulation is the result of a disproportionate decrease in reaction times on incongruent trials (see Table 1).

In contrast to right-hemisphere stimulation, rTMS over the left PPC had no effect on interference (see Figure 3; C.P.: $p=.22$, E.F.: $p=.09$ ). Thus, the disruption of synesthesia following right parietal rTMS does not generalize to the left-hemisphere homologue. This is consistent with neurophysiological evidence that the right parietal cortex plays a more critical role in normal color-form binding than does the left parietal cortex (Ashbridge et al., 1997).
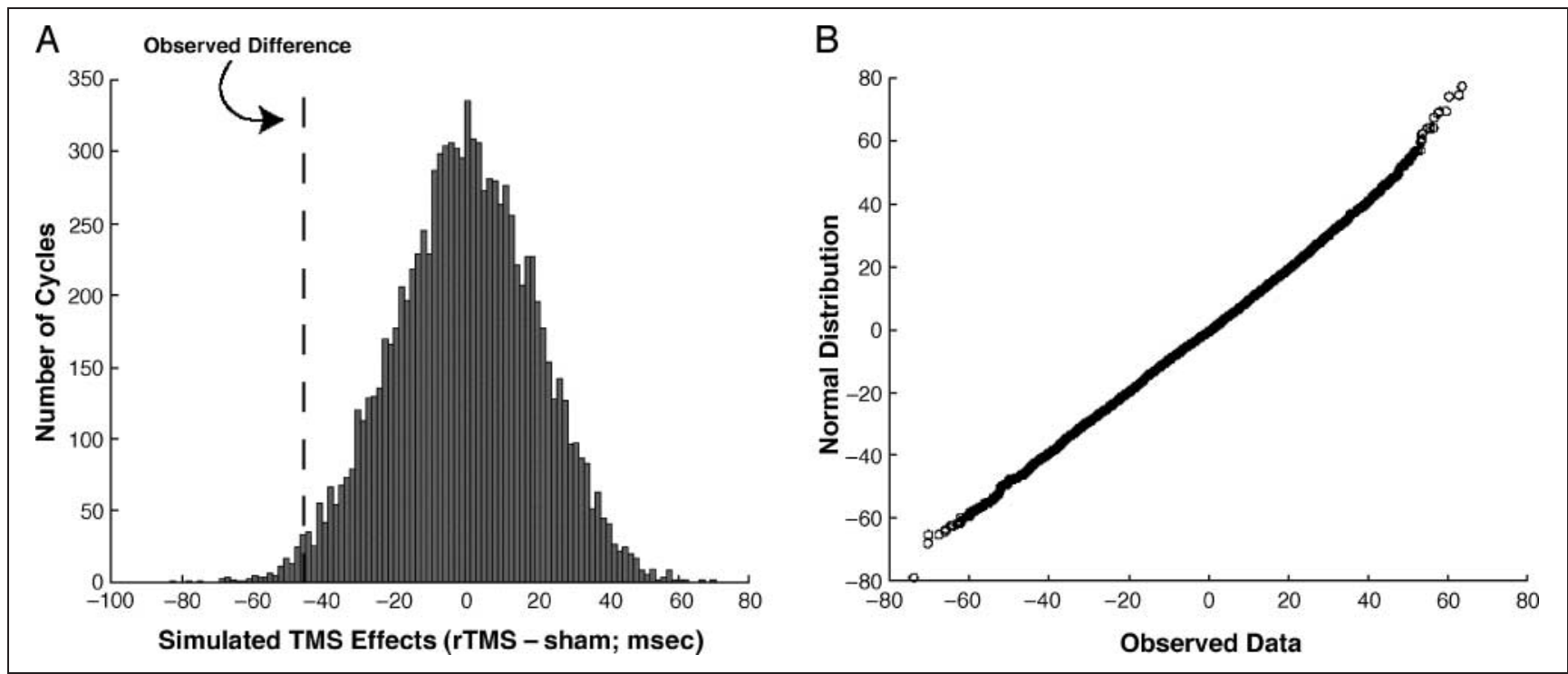

Figure 2. (A) A histogram of simulated TMS effects representing a hypothetical null distribution for C.P. after right PPC stimulation. The observed reaction times were randomly recategorized (with replacement) and new interference effects were calculated from these permutated data sets. This process was repeated 10,000 times to produce a distribution of values that represent what would be observed purely by chance. The dashed line represents the true observed TMS effect immediately following stimulation. (B) A normal probability plot, comparing the data presented in (A) with values from a normal distribution with mean of $-0.04 \mathrm{msec}$ and standard deviation of 20 msec. The approximately straight line indicates that the hypothetical null distribution reflect a normal gaussian process. 


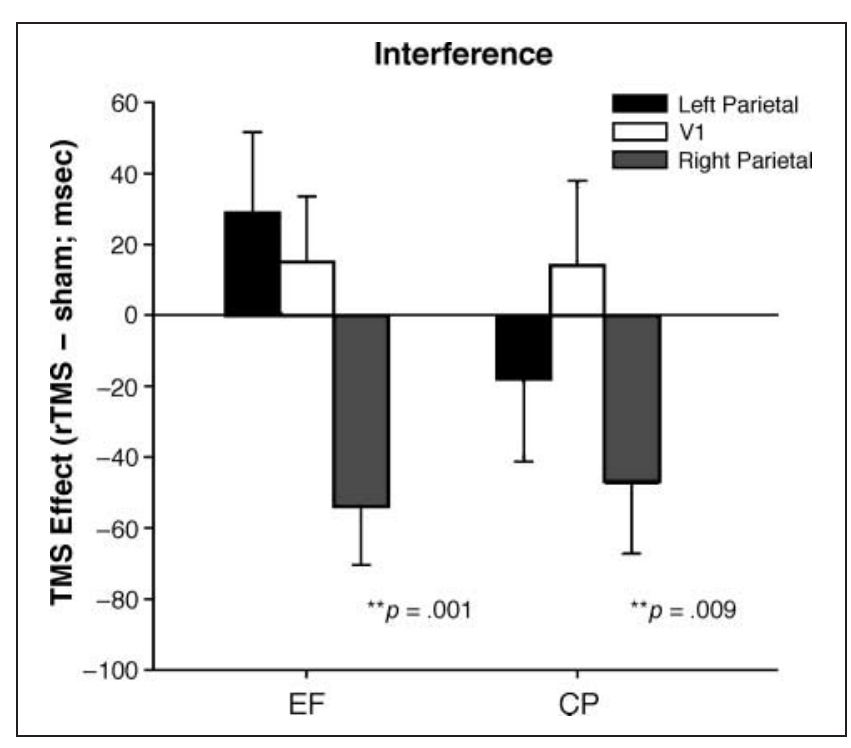

Figure 3. Effects of TMS on synesthetic interference. Graph displays effects of rTMS on synesthetic interference (incongruent minus neutral) for both participants immediately following stimulation (early block; see text). Both participants demonstrated a strong attenuation of interference following right parietal compared to sham rTMS, but no change following stimulation of the left parietal region or V1 (see Methods for discussion of statistical procedure and Table 1 for reaction times and standard deviations in all conditions).

rTMS over V1 also had no effect on interference (see Figure 3; C.P.: $p=.28$, E.F.: $p=.21$ ). Thus, the disruption of synesthesia following right parietal rTMS is not due to generalized arousal following stimulation and suggests that V1 does not play a critical role in color-form binding.

Unlike interference, facilitation was not reliably observed in our participants. Naming colors that were synesthetically congruent produced consistently faster responses for participant E.F only (36 msec). C.P. did not show consistent facilitation in the sham blocks (mean $=14 \mathrm{msec}$; range, -26 to $44 \mathrm{msec}$ ). Facilitation is not reliably observed in traditional Stroop tasks in normal observers (Tzelgov, Henik, \& Berger, 1992; MacLeod, 1991) as well as tasks similar to ours when tested in other synesthetes (Sagiv et al., 2006; Mattingley et al., 2001; Dixon et al., 2000). It has been proposed that facilitation, when observed in traditional Stroop tasks, may be due to inadvertent reading, which would explain our weak facilitation effect, given that "reading" the letter would not lead to correct responses in our paradigm (MacLeod, 1991). Our results are consistent with studies of normal perceivers showing that facilitation and interference rely on different mechanisms (Tzelgov et al., 1992).

In sum, immediately following rTMS of the right PPC, both participants showed a significant attenuation of interference normally induced by their synesthesia. We propose that right parietal rTMS produced a transient disruption of synesthetic color-form integration, thus reducing the conflict when the synesthetic photism did not correspond to the real color of the letter. These findings are consistent with evidence implicating the IPS/TOS in feature binding in normal perception (Donner et al., 2002; Friedman-Hill et al., 1995) and suggests that this region contributes to feature binding even when the perceived color is evoked by shape rather than wavelength (Robertson, 2003).

The present study supports the theory that feedback from a multimodal association region, like the parietal cortex, contributes to the perception of a synesthetic photism (Cohen-Kadosh \& Henik, 2006; Sagiv et al., 2006; Robertson, 2003; Grossenbacher \& Lovelace, 2001). Although this does not exclude the possibility that direct connections between fusiform regions also play a role in synesthesia (Ramachandran \& Hubbard, 2001), it suggests that cross wiring between these regions is not always sufficient to bind the synesthetic percept to the inducing character.

An alternative explanation for our findings is that parietal TMS attenuates competition between perceptually related codes, similar to competition produced between the perceptually and conceptually related codes in the traditional Stroop task where the color of the word may be congruent or incongruent with the word itself. According to this view, parietal TMS would interfere with competition between the color word and color (traditional Stroop) as well as competition between two colors, one induced by wavelength and the other by the letter (synesthesia), rather than interfere with feature binding per se. Although imaging studies have associated parietal activity with Stroop tasks (MacLeod \& MacDonald, 2000), it is unlikely that the parietal lobe plays a critical role in the competitive process underlying the traditional Stroop effect for several reasons. First, bilateral stimulation of the posterior parietal lobes does not influence the magnitude of Stroop interference or facilitation (Hayward, Goodwin, \& Harmer, 2004).

Second, neuropsychological studies of patients with parietal lesions have demonstrated normal Stroop interference compared to controls. Interestingly, this effect has been reported in patients with either left, right, or bilateral infarcts (Vivas, Humphreys, \& Fuentes, 2003; Robertson, Treisman, Friedman-Hill, \& Grabowecky, 1997; Berti, Frassinetti, \& Umilta, 1994), and these same patients may exhibit profound deficits in color-form binding (see Robertson, 2003). In contrast, alterations in Stroop performance have been associated with damage to the prefrontal cortex (Kato, 2001; Stuss, Floden, Alexander, Levine, \& Katz, 2001).

Third, the three studies that have implicated the parietal lobe in color-grapheme synesthesia have not involved Stroop tasks, indicating that the parietal lobe's role in synesthesia is not dependent on response interference (Weiss et al., 2005; Elias et al., 2003; Rich et al., 2003). Together, these different lines of research 
Table 1. Single-subject Reaction Times

\begin{tabular}{|c|c|c|c|c|c|c|}
\hline & \multicolumn{3}{|c|}{ Participant E.F. } & \multicolumn{3}{|c|}{ Participant C.P. } \\
\hline & Neutral & Congruent & Incongruent & Neutral & Congruent & Incongruent \\
\hline \multicolumn{7}{|c|}{ Right parietal } \\
\hline \multicolumn{7}{|c|}{ Early phase } \\
\hline Sham & $476 \pm 103$ & $424 \pm 77$ & $546 \pm 114$ & $615 \pm 161$ & $574 \pm 158$ & $647 \pm 186$ \\
\hline rTMS & $481 \pm 88$ & $423 \pm 58$ & $497 \pm 96$ & $550 \pm 145$ & $511 \pm 118$ & $535 \pm 94$ \\
\hline \multicolumn{7}{|c|}{ Late phase } \\
\hline Sham & $483 \pm 86$ & $453 \pm 97$ & $531 \pm 92$ & $548 \pm 108$ & $575 \pm 169$ & $597 \pm 185$ \\
\hline rTMS & $509 \pm 117$ & $441 \pm 80$ & $570 \pm 131$ & $591 \pm 166$ & $546 \pm 145$ & $601 \pm 184$ \\
\hline
\end{tabular}

\section{Left parietal}

Early phase

$\begin{array}{ccccccr}\text { Sham } & 492 \pm 120 & 434 \pm 81 & 523 \pm 116 & 503 \pm 99 & 503 \pm 104 & 544 \pm 152 \\ \text { rTMS } & 469 \pm 84 & 449 \pm 93 & 530 \pm 141 & 499 \pm 118 & 471 \pm 96 & 523 \pm 134 \\ \text { ate phase } & & & & & & \\ \text { Sham } & 496 \pm 90 & 462 \pm 94 & 529 \pm 119 & 533 \pm 113 & 521 \pm 111 & 557 \pm 132 \\ \text { rTMS } & 471 \pm 95 & 462 \pm 108 & 532 \pm 133 & 495 \pm 114 & 490 \pm 103 & 536 \pm 145\end{array}$

V1

Early phase

\begin{tabular}{lllllll} 
Sham & $452 \pm 76$ & $419 \pm 70$ & $485 \pm 91$ & $591 \pm 121$ & $547 \pm 141$ & $611 \pm 156$ \\
rTMS & $440 \pm 99$ & $412 \pm 62$ & $488 \pm 107$ & $511 \pm 106$ & $486 \pm 107$ & $544 \pm 144$ \\
ate phase & & & & & & \\
Sham & $423 \pm 75$ & $413 \pm 64$ & $514 \pm 113$ & $568 \pm 117$ & $556 \pm 168$ & $587 \pm 150$ \\
rTMS & $443 \pm 98$ & $418 \pm 74$ & $501 \pm 84$ & $524 \pm 127$ & $511 \pm 132$ & $529 \pm 152$ \\
\hline
\end{tabular}

Shown are mean reaction times and standard deviations across each condition (neutral, congruent, or incongruent), phase (early, late), participant (E.F., C.P.) and each stimulation site (right parietal, left parietal, V1).

strongly suggest that our effect is not due to response interference in general.

Behavioral and physiological evidence suggests that synesthesia is a heterogeneous phenomenon (Dixon \& Smilek, 2005; Hubbard et al., 2005). These individual differences may be related to the degree of parietal involvement. One behavioral factor that may contribute to this heterogeneity is the difference between projectors, those who see the synesthetic photism in the world, and associators, those who see the photism in the mind's eye (Dixon et al., 2004). Although this factor has not been explored with respect to the degree of parietal involvement, one hypothesis to consider in future research is that synesthetes who show greater parietal involvement may tend to be "projectors," particularly when the synesthetic percept is tightly bound spatially to the inducing grapheme. Physiologically, the degree of parietal contribution among synesthetes may also be greater in individuals such as C.P., in whom the extent of early visual activation has been shown to be lesser (see Hubbard et al., 2005). Parietal participation in the synesthetic experience may correspond to the extent that attention is required to elicit the photism. The variability in the expression of color-grapheme synesthesia makes the examination of individual differences vital to our understanding of this fascinating phenomenon (Dixon \& Smilek, 2005; Hubbard et al., 2005; Dixon et al., 2004). Our results have illuminated one of potentially several mechanisms responsible for synesthesia and are consistent with theories of how normal binding occurs within the human brain.

Future studies must examine the role of parietal binding mechanisms in other synesthetes, as well as investigate other parietal and temporal lobe regions that have been implicated in spatial attention and binding, such as the superior parietal cortex and the superior temporal 
sulcus, using both TMS and fMRI. In addition, single-pulse TMS will be able to elaborate on the temporal dynamics of the right PPC's involvement in synesthesia.

\section{Acknowledgments}

We thank Noam Sagiv for discussions and motivation that led to this project, Noam Sobel for comments on an early version of this manuscript, and Edward Hubbard for his constructive comments during the review process.

Reprint requests should be sent to Michael Esterman, Department of Psychology and Neuroscience Institute, University of California, Berkeley, CA, USA, or via e-mail: esterman@berkeley.edu.

\section{REFERENCES}

Ashbridge, E., Walsh, V., \& Cowey, A. (1997). Temporal aspects of visual search studied by transcranial magnetic stimulation. Neuropsychologia, 35, 1121-1131.

Berti, A., Frassinetti, F., \& Umilta, C. (1994). Nonconscious reading? Evidence from neglect dyslexia. Cortex, 30, 181-197.

Chambers, J., Cleveland, W., Kleiner, B., \& Tukey, P. (1983). Graphical methods for data analysis (Wadsworth). Boston: Duxbury Press.

Cohen-Kadosh, R., \& Henik, A. (2006). Color congruity effect: Where do colors and numbers interact in synesthesia? Cortex, 42, 259-263.

Dixon, M. J., \& Smilek, D. (2005). The importance of individual differences in grapheme-color synesthesia. Neuron, 45, 821-823.

Dixon, M. J., Smilek, D., Cudahy, C., \& Merikle, P. M. (2000). Five plus two equals yellow. Nature, 406, 365.

Dixon, M. J., Smilek, D., \& Merikle, P. M. (2004). Not all synaesthetes are created equal: Projector versus associator synaesthetes. Cognitive, Affective, and Behavioral Neuroscience, 4, 335-343.

Donner, T. H., Kettermann, A., Diesch, E., Ostendorf, F., Villringer, A., \& Brandt, S. A. (2002). Visual feature and conjunction searches of equal difficulty engage only partially overlapping frontoparietal networks. Neuroimage, 15, 16-25.

Elias, L. J., Saucier, D. M., Hardie, C., \& Sarty, G. E. (2003). Dissociating semantic and perceptual components of synaesthesia: Behavioral and functional neuroanatomical investigations. Cognitive Brain Research, 16, 232-237.

Epstein, C. M., \& Davey, K. R. (2002). Iron-core coils for transcranial magnetic stimulation. Journal of Clinical Neurophysiology, 19, 376-381.

Friedman-Hill, S., Robertson, L., \& Treisman, A. (1995). Parietal contributions to visual feature binding: Evidence from a patient with bilateral lesions. Science, 269, 853-855.

Grossenbacher, P. G., \& Lovelace, C. T. (2001). Mechanisms of synesthesia: Cognitive and physiological constraints. Trends in Cognitive Sciences, 5, 36-41.

Hayward, G., Goodwin, G. M., \& Harmer, C. J. (2004). The role of the anterior congulate cortex in the counting Stroop task. Experimental Brain Research, 154, 355-358.

Hubbard, E. M., Arman, A. C., Ramachandran, V. S., \& Boynton, G. M. (2005). Individual differences among grapheme-color synesthetes: Brain-behavior correlations. Neuron, 45, 975-985.

Hubbard, E. M., \& Ramachandran, V. S. (2005). Neurocognitive mechanisms of synesthesia. Neuron, 48, 509-520.

Kato, M. (2001). Prefrontal lobes and the attentional control: A neuropsychological study using modified Stroop test. Rinsho Shinkeigaku, 41, 1134-1136.
MacLeod, C. M. (1991). Half a century of research on the Stroop effect: An integrative review. Psychological Bulletin, 109, 163-203.

MacLeod, C. M., \& MacDonald, P. A. (2000). Interdimensional interference in the Stroop effect: Uncovering the cognitive and neural anatomy of attention. Trends in Cognitive Sciences, 4, 383-391.

Manly, B. (1997). Randomization, bootstrap and Monte Carlo methods in biology (2nd ed.). New York: Chapman and Hall/CRC.

Mattingley, J. B., Rich, A. N., Yelland, G., \& Bradshaw, J. L. (2001). Unconscious priming eliminates automatic binding of colour and alphanumeric form in synaesthesia. Nature, 410, 580-582.

Palmeri, T. J., Blake, R., Marois, R., Flanery, M. A., \& Whetsell, W. (2002). The perceptual reality of synesthetic colors. Proceedings of the National Academy of Sciences, U.S.A., 99, 4127-4131.

Pascual-Leone, A., Tormos, J. M., Keenan, J., Tarazona, F., Canete, C., \& Catala, M. D. (1998). Study and modulation of human cortical excitability with transcranial magnetic stimulation. Journal of Clinical Neurophysiology, 15, 333-343.

Ramachandran, V. S., \& Hubbard, E. M. (2001). Psychophysical investigations into the neural basis of synaesthesia. Proceedings of the Royal Society of London, Series B, Biological Sciences, 268, 979-983.

Rich, A. N., \& Mattingley, J. B. (2002). Anomalous perception in synaesthesia: A cognitive neuroscience perspective. Nature Reviews, 3, 43-52.

Rich, A. N., Puce, A., Syngeniotis, A., Williams, M. A., Howard, M. A., McGlone, F., \& Mattingley, J. B. (2003). Colour my brain: A functional neuroimaging study of color-graphemic synaesthesia. Paper presented at the annual meeting of the Cognitive Neuroscience Society, New York, NY.

Robertson, L., Treisman, A., Friedman-Hill, S., \& Grabowecky, M. (1997). The interaction of spatial and object pathways: Evidence from Balint's syndrome. Journal of Cognitive Neuroscience, 9, 295-317.

Robertson, L. C. (2003). Binding, spatial attention and perceptual awareness. Nature Reviews Neuroscience, 4, 93-102.

Sagiv, N., Heer, J., \& Robertson, L. C. (2006). Does binding of synesthetic color to the evoking grapheme require attention? Cortex, 42, 232-242.

Smilek, D., Dixon, M. J., \& Merikle, P. M. (2004). Binding of graphemes and synesthetic colors in color-graphemic synesthesia. In N. Sagiv \& L. Robertson (Eds.), Synesthesia: Perspectives from cognitive neuroscience. New York: Oxford University Press.

Sperling, J. M., Prvulovic, D., Linden, D. E. J., Singer, W., \& Stirn, A. (2006). Neuronal correlates of colour-graphemic synaesthesia: A fMRI study. Cortex, 42, 295-303.

Stuss, D. T., Floden, D., Alexander, M. P., Levine, B., \& Katz, D. (2001). Stroop performance in focal lesion patients: Dissociation of processes and frontal lobe lesion location. Neuropsychologia, 39, 771-786.

Tzelgov, J., Henik, A., \& Berger, J. (1992). Controlling Stroop effects by manipulating expectations for color words. Memory \& Cognition, 20, 727-735.

Vivas, A. B., Humphreys, G. W., \& Fuentes, L. J. (2003). Inhibitory processing following damage to the parietal lobe. Neuropsychologia, 41, 1531-1540.

Weiss, P. H., Zilles, K., \& Fink, G. R. (2005). When visual perception causes feeling: Enhanced cross-modal processing in grapheme-color synesthesia. Neuroimage, 28, 859-868. 